\title{
Management of high-risk popliteal vascular blunt trauma: clinical experience with 62 cases
}

\author{
This article was published in the following Dove Press journal: \\ Vascular Health and Risk Management \\ 10 July 2010 \\ Number of times this article has been viewed
}

\section{Ali Pourzand \\ Bassir A Fakhri \\ Ramin Azhough \\ Mohammad Ali Hassanzadeh \\ Shahryar Hashemzadeh \\ Amrollah M Bayat}

Department of General Surgery,

Tabriz University of Medical Sciences,

Tabriz, East Azarbaijan Province, Iran
Correspondence: Ramin Azhough Department of General Surgery, Azadi Street, Emam Reza hospital, Tabriz, Iran $\mathrm{Tel}+989143113249$

Fax +98 4II 334 I3 I7

Email azhough@yahoo
Purpose: The purpose of this study is to report the clinical and functional outcomes of patients, treated between 2004 and 2009, with high-risk popliteal vascular injuries due to compound fractures about the knee.

Patients and methods: A retrospective analysis was conducted of prospectively collected data from Tabriz Medical Trauma Center. Our aim was to perform surgical revascularization as soon as the arterial injury was recognized. The mechanism of injury was blunt in the entire cohort of patients, and all of them had bone fractures about the knee. The treatment of arterial injury included vein graft interposition in 39 (63\%), primary anastomosis in $20(32.3 \%)$, and lateral repair in $3(4.8 \%)$ patients. The patients were divided into 2 study groups: limb salvage group (group 1) and amputation group (group 2). Subgroup analysis consisted of univariate analysis comparing the 2 groups and multivariate analysis examining the factors associated negatively and positively with the primary endpoint, limb salvage.

Results: In the entire cohort of patients, 60 patients (97\%) were male and 2 were female (3\%); the mean age was 34.1 years (16-49 years). The overall amputation rate in this study was $37.1 \%$ (23 amputations). Significant $(P<0.05)$ independent factors associated negatively with limb salvage were combined tibia and fibula fracture, concomitant artery and vein injury, ligation of venous injury, and lack of backflow after Fogarty catheter thrombectomy, while repair of popliteal artery and vein injury, when present, was associated with improved early limb salvage. For 40 patients, we adopt a liberal attitude toward open 4-compartment fasciotomy through both medially and laterally placed incisions.

Conclusion: Expeditious recognition of vascular injury, transport to repair, and repair of associated venous injury when possible are necessary to optimize limb salvage. The importance of a high level of suspicion and low threshold for timely amputation has been emphasized when limb salvage was deemed impossible to prevent life-threatening complication. Delays in surgery, extensive soft tissue defect, compound tibia-fibula fracture, and other factors are associated with high amputation rate following popliteal artery injury.

Keywords: popliteal vascular blunt trauma, knee, fracture

\section{Introduction}

Injury to the popliteal vessels has been recognized as one of the most limb-threatening peripheral vascular injuries as long as vascular trauma has been studied. The popliteal artery is a true end artery with a tenuous collateral supply. The popliteal vein provides the bulk of lower leg and foot drainage. This explains why injury to these vessels is so dangerous. ${ }^{1}$ Extremity vascular trauma is often associated not only with the brain and torso injuries but also with local bony and soft tissue defects. Management of these multiple-injured patients can be very challenging, as restoring perfusion to an ischemic 
extremity needs to be accomplished expeditiously, generally within 6-8 hours, if function is to be salvaged.

The traditional wartime surgical experience has led to advances in the treatment of traumatic vascular injury. Military operations in support of Operation Iraqi Freedom represent the first mature military conflict since Vietnam War to allow assessment of contemporary practices such as vascular shunts in the management of wartime vascular injuries. This experience supports the early detection of the injured vessel(s) and use of shunts in certain cases as part of an overall strategy in the management of vascular injury. ${ }^{2,3}$ Early results of in-theater repair are comparable with contemporary civilian reports and have greatly improved from the Vietnam era. Rapid evacuation and damage control maneuvers, such as temporary shunting and early fasciotomy, assist in timely definitive repair and appear effective. ${ }^{4}$ The purpose of this study is to report the management techniques and factors associated with early limb salvage following civilian popliteal vascular injury managed at an Iranian trauma center. Additionally, controversial aspects of managing such injuries are examined.

\section{Patients and methods}

\section{Patient cohort and data collection}

From July 2004 to July 2009, 62 patients had arterial reconstruction for acute traumatic injuries to popliteal arteries associated with bone injuries about the knee, at Imam Reza Hospital in Tabriz Medical Trauma Center, by the most senior resident of general surgery under direct supervision of full-time attending surgeons. This center is a busy trauma unit located in northwest of Iran, in an area with high rates of inflicted and accidental injuries. The unit receives referrals from the province of East Azarbaijan and occasionally from surrounding provinces and involves nearly 100 level II and III centers. In this study, all patients were transported by ground. Patients with primary traumatic limb amputations, injuries to the iliac or proximal femoral vessels, gunshot injuries, penetrating wounds, and popliteal vessel injuries without fracture were excluded from this study. Primary amputations were defined as those in which no attempt at limb salvage was made, and removal of leg was performed as the initial procedure. All amputations in this report are defined as secondary amputations as they were performed following an initial attempt at revascularization and limb salvage. Each patient's hospital course was reviewed retrospectively for signs and symptoms of vascular compromise from admission until discharge. The presence of hard signs of arterial injury (active hemorrhage; large, expanding, or pulsatile hematoma; bruits or thrills; absence of pulse; and distal ischemia) was considered an indication for surgery, and no further specific investigative measures were taken. Data collected from the medical record included demographic information, location of injury (ie, right or left lower extremity), associated torso or traumatic brain injury, physiologic indicators of shock, concomitant popliteal venous injury, transfusion requirements, and time from injury to arrival at Tabriz Trauma Center.

\section{Operative technique}

After initial resuscitation and assessment, patients with confirmed arterial injury were operated on as soon as possible under general anesthesia. Exposure was made through an incision in the medial aspect of knee with the approximate site of injury at its center. After the affected arterial segment was controlled, an embolectomy catheter was passed distally and proximally, and the backflow and inflow were assessed. If arterial grafting was required, reversed contralateral greater saphenous vein grafts were used. The insertion of arterial shunts at this stage was not used. After thrombectomy, the arterial segments were liberally flushed with heparinized saline. The arterial injuries were repaired before orthopedic injuries. After orthopedic management, graft visualization was clinically reassessed by the surgeon to ensure the integrity of repair before reversal of anesthesia. If simple venous injury was found, it was repaired by primary suture; however, the complex injuries such as those requiring vein grafting were ligated. For partial nerve transection, primary repair was carried out. When complete nerve transection was observed, nerve endings were identified and marked for delayed secondary repair. The major endpoint was limbsalvage rate of the patients.

\section{Statistical analysis}

Subgroup analysis was performed by dividing the overall cohort into 2 study groups: Group 1 (limb salvage; $\mathrm{N}=39$ ) and group 2 (amputation; $\mathrm{N}=23$ ). An analysis was performed comparing groups 1 and 2, and the factors found to be different between the groups were entered into a stepwise multivariate regression analysis to determine those associated positively and negatively with the endpoint of early limb salvage.

Data were compared with independent Student $t$ tests or one-way analysis of variance, and categorical proportions with contingency table with chi-square test or Fisher exact test, as appropriate. Stepwise logistic regression was 
used to examine the independent associations of various demographic and injury-related factors. $P$ was assumed to be significant if less than or equal to 0.05 in all tests. Statistical analysis was performed with SPSS ${ }^{\mathrm{TM}} 13$ software.

\section{Results}

In the entire cohort of patients, 60 patients $(97 \%)$ were male and 2 were female ( $3 \%$ ); the mean age was 34 years (16-49 years). Trauma resulted from motorcycle accidents in $33(53.2 \%)$ cases, car accidents in $24(38.7 \%)$, and fall from a high level in $5(8 \%)$.

In all cases, no distal pulse was palpable at the time of admission. The mean length of followup among the limb salvage group was 16 months (range, 1-34 months; Table 1). Treatment of arterial injury included vein graft interposition in $39(63 \%)$, primary anastomosis in $20(32.3 \%)$, and lateral repair in $3(4.8 \%)$.

The overall amputation rate in this study was $37 \%$ (23 secondary amputations). Primary amputation was performed in 2 patients: 1 for severe crush injury and the other for extensive injury following bomb explosion. The amputation level was above the knee in 20 cases and below the knee in 3 cases. All of these amputations were performed when limb salvage was deemed impossible in the course of hospital stay. Table 1 shows univariate analysis of factors related to early limb salvage.

All patients in this study had some form of skeletal injury. The presence of combined tibia-fibula fracture and arterial injury was negatively associated with early limb salvage [odds ratio $(\mathrm{OR})=0.27$; confidence interval $(\mathrm{CI})$, 0.09-0.81]; however, combined femur and tibial fracture and isolated tibial fracture alone had no association with early limb salvage.

Associated remote injuries were found in 74\% and $62 \%$ of patients in the amputee and salvage groups, respectively. Mean number of associated remote injuries was associated negatively with limb salvage $(1.08 \pm 0.90$ in amputee and $0.64 \pm 0.53$ in salvage group, $\mathrm{OR}=0.57$; CI, 0.18-1.75), but distinct type of remote injury had no association with early limb salvage.

The presence of sensory or motor dysfunction at the time of presentation displayed a negative association with early limb salvage $(\mathrm{OR}=0.05$; $\mathrm{CI}, 0.01-0.30)$.

The lack of backflow after thrombectomy was a poor sign and was associated negatively with limb salvage $(\mathrm{OR}=0.15$; CI, 0.01-0.18). Concomitant venous injury was negatively associated with early limb salvage $(\mathrm{OR}=0.14$; CI, 0.03-0.46) as was venous ligation ( $\mathrm{OR}=0.5$; CI, 0.005-0.42).

Although it was difficult to obtain accurate details regarding time interval between injury and hospital admission for all patients, mean time of transport in our area was 6 hours. Increased delay had adverse effect on limb salvage rate $(\mathrm{OR}=0.07$; CI, 0.001-0.08).

Physiologic triggers are accurate predictors of transfusion requirements as they are based on the patient's specific needs with respect to deranged homodynamic in primary patients' management. Transfusion of more than $10 \mathrm{U}$ of packed cells due to nearby or remote injury bleeding was associated negatively with limb salvage rate $(\mathrm{OR}=0.10$; CI, 0.01-0.87).

Forty patients received open 4-compartment fasciotomy using 2 incision (medial and lateral) approaches. Thirty-six

Table I Univariate analysis of different factors associated with early limb salvage

\begin{tabular}{|c|c|c|c|c|}
\hline Study variable & OR $(95 \% \mathrm{CI})$ & $\begin{array}{l}\text { Amputee group } \\
\text { ( } 23 \text { cases) }\end{array}$ & $\begin{array}{l}\text { Salvage group } \\
\text { ( } 39 \text { cases) }\end{array}$ & $P$ \\
\hline Femur fracture & $4(0.45-35.55)$ & I (4.3\%) & $6(15.4 \%)$ & 0.24 \\
\hline Tibia-fibula (double fracture) & $0.27(0.09-0.8 \mathrm{I})$ & $16(69.6 \%)$ & $15(38.5 \%)$ & $0.018^{\mathrm{a}}$ \\
\hline Femur and tibia & $0.86(0.21-3.45)$ & $4(17.4 \%)$ & $6(15.4 \%)$ & 1 \\
\hline Knee joint fracture with dislocation & $3.62(0.7 I-18.27)$ & $2(8.7 \%)$ & $10(25.6 \%)$ & 0.18 \\
\hline Isolated tibia fracture & $1.05(0.10-13.88)$ & 0 & $2(5.1 \%)$ & 0.52 \\
\hline Extensive soft tissue defect & $0.02(0.003-0.24)$ & II (47.8\%) & I (2.6\%) & $0.000 I^{\mathrm{a}}$ \\
\hline Concomitant popliteal artery and vein injury & $0.14(0.3-0.46)$ & $9(39.1 \%)$ & II (28.2\%) & $0.044^{a}$ \\
\hline Artery repair and popliteal vein ligation & $0.49(0.005-0.42)$ & $8(34.8 \%)$ & $\mathrm{I}(2.6 \%)$ & $0.0009^{a}$ \\
\hline Artery and popliteal vein repair & $7.58(0.90-63.77)$ & I (4.3\%) & $10(26 \%)$ & $0.042^{\mathrm{a}}$ \\
\hline Lack of backflow after thrombectomy & $0.15(0.01-0.18)$ & $18(78.2 \%)$ & $6(15.3 \%)$ & $<0.000 I^{\mathrm{a}}$ \\
\hline Delay before reaching the hospital $>6 \mathrm{~h}$ & $0.07(0.00 I-0.08)$ & $22(95.7 \%)$ & $7(18 \%)$ & $<0.003^{a}$ \\
\hline Packed cell transfusion $>10 \mathrm{U}$ & $0.10(0.01-0.87)$ & $5(21.7 \%)$ & I (2.6\%) & $0.023^{\mathrm{a}}$ \\
\hline Fasciotomy & $0.70(0.23-2.09)$ & $16(69.6 \%)$ & $24(61.5 \%)$ & 0.523 \\
\hline Pulseless, but perfusion normal & $18.8(2.30-154.08)$ & I (4.3\%) & $18(46.2 \%)$ & $0.0004^{a}$ \\
\hline Pulseless, cool, insensate, numb & II (47.8) & $2(5.1 \%)$ & $0.05(0.01-0.30)$ & $0.000 I^{a}$ \\
\hline
\end{tabular}

ap $<0.05$ significant, significant variables. 
fasciotomies were performed early before or immediately following vascular repair, and 4 were performed in a delayed fashion. The diagnosis of compartment syndrome based on clinical suspicion, delay of more than 6 hours, severe extremity ischemia, and popliteal vein ligation were selection criteria for fasciotomy in our experiments. The fasciotomy wounds were irrigated regularly and necrotic tissues, if any, excised periodically until the wounds were clean and acceptable for closure.

Of all patients studied, 7 died during their hospital stay. The causes of death were sepsis in 3 and multiorgan failure in another 3 patients. One of our patients was victim to a prescription error.

Twenty-three patients (65.7\%) had a useful and functional limb following repair and were generally pleased with the result. They could walk in 2 months without assistance. The complications included peroneal nerve palsy in 7 patients. Three of these patients recovered completely after 6 months of rehabilitation. In 4 patients, peripheral nerve deficit remained unchanged at 14 months. Five patients had tibial nerve injury. Two of them had partial nerve injury on operation, so primary repair was performed and recovered partially at 26 months and benefited from the muscle tendon transfer surgery, providing sufficient ankle strength and mobility to ambulate without a brace. In 3 patients, tibial nerve deficit remained unchanged at 18 months after secondary nerve repair.

A multivariate logistic regression model was created to identify factors independently associated with early limb salvage. In this analysis, 11 preoperative, intraoperative, and postoperative variables were entered. Five factors were identified to be independently (negatively) associated with limb salvage. These were concomitant vein injury; popliteal vein ligation; tibia-fibula fracture; pulseless, cool, and insensate limb at presentation; and lack of backflow after catheter thrombectomy.

In contrast, the repair of concomitant popliteal artery and vein injury when present had a positive association with limb salvage. Table 2 shows the relative risks and $P$-values for these variables.

\section{Discussion}

Experience during World War II demonstrated that simple ligation of the popliteal artery results in $73 \%$ amputation rate. ${ }^{5}$ The management of complex injuries involving vascular and skeletal elements of the lower extremity remains challenging and still incurs a high incidence of limb loss and morbidity. Admittedly, the variable approaches and results reported in the literature reflect the heterogeneity of these injuries. The overriding principle in treating acute traumatic arterial injuries to the lower extremity is to avoid prolonged warm ischemia. This reduces the risk of both irreversible ischemia and morbidity associated with ischemia reperfusion. ${ }^{6}$ The situation regarding time interval between injury and admission raises unique problems in our area for reasons of size and a sparse population and limited number of centers that are able to manage these cases. Our experience shows that duration of ischemia plays a central role in morbidity, especially when it is over 6 hours. Although the time interval between injury and arrival at trauma center rarely is controlled by the surgeon, the time interval between arrival at the hospital and operation is under the surgeon's control. Immediate exploration without angiography should be undertaken for patients with more severe ischemia or active hemorrhage from the suspected artery when any delay in intervention could threaten the limb and/or the life of the patient. On-table angiography performed in the operating suite before exploration is quicker and is advised if the specific site of injury is in doubt. ${ }^{7}$ Odland et $\mathrm{al}^{8}$ reported that shock and crush injuries are associated with a significant rate of amputation.

In each of our cases, vascular repair preceded orthopedic intervention. It is, however, easy to appreciate that subsequent orthopedic surgery on a limb, which may be mechanically unstable, may compromise a successful vascular repair. There have been various solutions to this problem. Most support the concept of priority of revascularization as restitution of

Table 2 Multivariate regression analysis of factors associated negatively and positively with early limb salvage

\begin{tabular}{lll}
\hline Study variable & OR (95\% Cl) & $P$ \\
\hline Tibia-fibula (double fracture) & $0.133(0.01-\mathrm{I} .0 \mathrm{I})$ & $0.032(\mathrm{n})$ \\
Artery repair and popliteal vein ligation & $0.326(0.16-0.66)$ & $0.002(\mathrm{n})$ \\
Concomitant artery and vein injury & $0.039(0.004-0.40)$ & $0.007(\mathrm{n})$ \\
Lack of backflow after catheter thrombectomy & $0.129(0.02-0.72)$ & $0.020(\mathrm{n})$ \\
Pulseless, cool, insensate, numb (at presentation) & $0.12(0.03-0.45)$ & 0.00 I (n) \\
Pulseless, but perfusion normal (at presentation) & $2.25(1.30-15.07)$ & $0.005(\mathrm{p})$ \\
Artery and popliteal vein repair & $2.8 \mathrm{I}(2.22-6.53)$ & $0.033(\mathrm{p})$ \\
\hline
\end{tabular}

Note: $n$, negatively associated with limb salvage; $p$, positively associated with limb salvage. 
blood flow is the most urgent demand. We prefer the vascular surgeon to remain in attendance to prevent excessive movement of the vascular repair during fracture reduction and stabilization. This is our general institutional policy. We did not have any loss of flow during orthopedic manipulation. An alternative immediate vascular reconstruction is the use of temporary vascular shunts to restore perfusion before orthopedic fixation. This vascular adjunct represents a safe and effective damage-control technique and is preferable to attempted reconstruction in austere conditions. Shunts in the arterial position allow for perfusion of the extremity during transport or fixation of associated orthopedic injuries. For injury patterns involving an artery and vein, shunts placed in the venous position provide drainage and decrease venous hypertension that can compound tissue ischemia and bleeding. ${ }^{3}$ Despite the reported utility of this technique, none of the patients in this series were managed with use of temporary vascular shunts.

Associated popliteal vein injury is another factor that can affect limb viability. The practice of venous ligation, as in the Vietnam War, has been shown to be associated with high amputation rates. Vein ligation can also lead to limb-threatening complications, including compartment syndrome, disabling edema, and failed arterial repair, unless life-threatening conditions coexist or when a more complex repair than simple suture or end-to-end anastomosis is necessary. ${ }^{7}$ Our practice has been to use simple repair and, if this proves impossible, ligation. As we see in Table 2, popliteal vein ligation was negatively associated with early limb salvage $(\mathrm{OR}=0.32$; CI, 0.16-0.66). In contrast, repair of popliteal vein injury had a positive association with limb salvage $(\mathrm{OR}=2.81$; CI, 2.22-6.53), but it should be considered with caution because of small sample size. The common thread among prolonged ischemia, severe injury to the soft tissues, combined injury to artery and vein, and compartment syndrome is macrovascular and microvascular thrombosis. Balloon-tipped catheters are relatively effective when used to remove thrombus from large vessels, but obviously are useless when considering the microvasculature. ${ }^{9}$ In our experience, lack of backflow after thrombectomy was seen in $18(78.2 \%)$ patients of amputee group compared with $6(15.3 \%)$ in limb-salvage group and was negatively associated with early limb salvage $(\mathrm{OR}=0.12$; $\mathrm{CI}, 0.02-0.72)$. Because most of our patients were young adults with no chronic peripheral vascular disease, we always expected restoration of at least 1 ankle pulse after operation. Graft failure occurred in some of our patients in limb salvage group, with the patients remaining asymptomatic because of excellent collateral circulation.

In this series, we had a relatively high rate of fasciotomy (64.5\%). More than one-third of these were absolutely indicated because of the presence of compartment syndrome on admission based on clinical finding of tense calf swelling. Remaining patients who received fasciotomy had the procedure immediately after revascularization when there was suspected or anticipated compartment syndrome. Only 4 patients had interval fasciotomy because of development of compartment syndrome related to ischemia-reperfusion injury later. It is generally agreed that early prophylactic fasciotomy is far better than therapeutic fasciotomy performed after the development of signs and symptoms of tissue compromise or tissue loss. ${ }^{1}$ Popliteal arterial injuries associated with bone injuries have a high amputation rate. Such injuries usually involve venous and soft tissue components. The rate of amputation decreased, thanks to the developments in shock therapy, use of antibiotics, better bone stabilization by external fixators, treatment of bone defects by internal bone transport, soft tissue reconstruction such as local and free flap, and rapid transportation. The amputation rate seems to be higher in blunt trauma than in penetrating trauma due to the fact that the former involves greater damage to the bone and soft tissue. ${ }^{8}$ Our results confirm that patients with injuries who present with frank ischemia or active hemorrhage and shock have a poorer prognosis and demand more urgent management than do patients with lesser injuries in which perfusion remains intact. Death due solely to peripheral vascular injuries is uncommon but does occur due to exsanguination or development of a necrotizing myofascial infection. We had 7 deaths in our series, 3 after amputation and 4 after limb salvage. Unfortunately, heroic salvage attempts in 2 of our cases led to catastrophic results. Extensive debridement of the devitalized soft tissues was done repeatedly, and our team's decision was to attempt limb salvage. After the first cycle, the electrolytes were maintained but serum creatinine rose. Urgent above-knee amputation was done when the patients became febrile septic. Most of the muscles in the thigh, which appeared viable and was actively bleeding during the primary surgery, were found nonviable and only very little muscle was salvaged, and above-knee amputation was performed. The second cycle continued during which the patient developed cardiac arrest with multiorgan failure from which he could not be resuscitated. The importance of a high index of suspicion and low threshold for timely amputation to prevent this uncommon yet life-threatening complication is, thus, emphasized again. 


\section{Conclusion}

Extensive injury to the vessels and surrounding structures complicates the management of blunt injuries and likely explains the higher amputation rates compared with penetrating injury. Not surprisingly, we found an associated significant trend toward worse functional outcome for blunt trauma patients. This was more remarkable in light of the fact that civilian vascular trauma includes a substantial portion of blunt injuries with their poorer overall prognosis. Patients who sustain popliteal vascular injuries need immediate transport to repair, rapid assessment and diagnosis at presentation, and revascularization. From the results obtained, it has been concluded that decreasing the severity of ischemia by fasciotomy, not wasting time with preoperative imaging and/or diagnostic tests, performing vascular repair before orthopedic repair in delayed cases, and repairing associated venous injury when possible are necessary to optimize limb salvage rates. The importance of a high index of suspicion and low threshold for timely amputation in the course of hospital stay when limb salvage was deemed impossible for the prevention of life-threatening complication has also been emphasized in this study.

\section{Disclosure}

The authors report no conflicts of interest in this work.

\section{References}

1. Frykberg ER. Popliteal vascular injuries. Surg Clin North Am. 2002;82.N;1.

2. Subramanian A, Vercruysse G, Dente Ch, Wyrzykowski A, Erin K, David VF. A decade's experience with temporary intravascular shunts at a civilian level I trauma center. J Trauma. 2008;65:316-326.

3. Rasmussen TE, Clouse WD, Jenkins DH, Peck MA, Eliason JL, Smith DL. The use of temporary vascular shunts as a damage control adjunct in the management of wartime vascular injury. J Trauma. 2006;61:8-15.

4. Woodward EB, Clouse WD, Eliason JL, Peck MA, Bowser AN, Cox MW. Penetrating femoropopliteal injury during modern warfare: experience of the Balad Vascular Registry. J Vasc Surg. 2008;47:1259-1264.

5. Klineberg EO, Crites BM, Flinn WR, Archibald JD, Moorman CT. The role of arteriography in assessing popliteal artery injury in knee dislocation. J Trauma. 2004;56:786-790.

6. Tam TT Huynh, Mai P, Lance WG, et al. Management of distal femoral and popliteal arterial injuries: an update. Am J Surg. 2006;192: 773-778.

7. Mazri MY, Bibombe PM, Manzoor A, Suhakar R, Kishore S. Popliteal artery injury: Royal Perth experience and literature review. ANZ J Surg. 2005;75: 882-886.

8. Subasi M, Cakir O, Kesemenli C, Arsalan H, Necmioglu S, Eren N. Popliteal artery injuries associated with fractures and dislocations about the knee. Acta Orthop Belg. 2001;67:3.

9. Melton SM, Croce MA, Patton JH, et al. Popliteal artery trauma. Systemic anticoagulation and intraoperative thrombolysis improves limb salvage. Ann Surg. 1997;225:518-529.
Vascular Health and Risk Management

\section{Publish your work in this journal}

Vascular Health and Risk Management is an international, peerreviewed journal of therapeutics and risk management, focusing on concise rapid reporting of clinical studies on the processes involved in the maintenance of vascular health; the monitoring, prevention and treatment of vascular disease and its sequelae; and the involvement of

\section{Dovepress}

metabolic disorders, particularly diabetes. This journal is indexed on PubMed Central and MedLine. The manuscript management system is completely online and includes a very quick and fair peer-review system, which is all easy to use. Visit http://www.dovepress.com/ testimonials.php to read real quotes from published authors. 